\title{
Transatlantica
}

Revue d'études américaines. American Studies Journal

\section{Smoke (Paul Auster et Wayne Wang, 1995) : une œuvre à la croisée des arts}

\section{Delphine Letort}

\section{(2) OpenEdition}

1 Journals

Édition électronique

URL : https://journals.openedition.org/transatlantica/5113

DOI : 10.4000/transatlantica.5113

ISSN : 1765-2766

Éditeur

Association française d'Etudes Américaines (AFEA)

Référence électronique

Delphine Letort, «Smoke (Paul Auster et Wayne Wang, 1995) : une œuvre à la croisée des arts », Transatlantica [En ligne], 2 | 2010, mis en ligne le 13 avril 2011, consulté le 02 février 2023. URL : http:// journals.openedition.org/transatlantica/5113; DOI : https://doi.org/10.4000/transatlantica.5113

Ce document a été généré automatiquement le 2 février 2023

\section{(c)}

Creative Commons - Attribution - Pas d'Utilisation Commerciale - Pas de Modification 4.0 International - CC BY-NC-ND 4.0

https://creativecommons.org/licenses/by-nc-nd/4.0/ 


\section{Smoke (Paul Auster et Wayne Wang, 1995) : une œuvre à la croisée des arts}

Delphine Letort

\section{NOTE DE L'AUTEUR}

Cet article a été l'occasion de riches échanges avec les correcteurs de Transatlantica. Sa conception illustre parfaitement le propos du film : un travail qui se nourrit de l'expérience de l'Autre. Je remercie vivement la générosité des correcteurs, dont les conseils avisés ont trouvé écho dans les lignes qui suivent.

1 Réalisé à partir d'un scénario original dont Paul Auster est l'auteur, Smoke représente la première aventure cinématographique de l'écrivain, qui accepta de mettre sa plume à l'épreuve de la caméra à la demande de Wayne Wang. Le réalisateur sollicita Paul Auster quelques semaines après la publication dans le New York Times d'une nouvelle qui avait immédiatement retenu son regard de cinéaste - « Auggie Wren's Christmas Story " (1990). L'histoire d'Auggie Wren nous est rapportée par un narrateur écrivain qui, contacté par l'éditeur du New York Times pour l'écriture d'un conte de Noël, ne parvient pas à surmonter l'angoisse de la page blanche. Seules les paroles échangées avec Auggie Wren lui permettront de renouer avec l'écriture et de satisfaire la commande qu'il s'était engagé à honorer. La magie de Noël traverse le conte de Paul Auster, qui offre son écriture à une histoire dont il suggère à travers l'ambiguïté d'une narration à la première personne qu'elle lui fut donnée, entretenant volontiers la confusion entre le réel et son appropriation dans la fiction. ${ }^{1}$ La nouvelle interroge le processus de création littéraire, qui se nourrit de l'expérience et du réel, dans un subtil jeu de rencontres et d'échanges entre l'écrivain (le narrateur) et le conteur/ photographe (Auggie Wren). 
Contrarié par d'autres projets d'écriture et de publication, notamment ceux des deux opus Leviathan (1992) et Mr Vertigo (1994), il fallut attendre 1993 pour que Paul Auster achève le scénario demandé par Wayne Wang, porté à l'écran à l'issue d'une série d'échanges entre les deux artistes en 1995. Le générique de Smoke présente à dessein le film comme le fruit d'une collaboration étroite entre Paul Auster et Wayne Wang, dont les modes d'expression s'entremêlent pour atteindre un compromis entre l'art de l'écriture et sa mise en image. Smoke prolonge le questionnement artistique qui soustend le récit de la nouvelle en interrogeant les modalités de l'appropriation du réel dans un autre mode artistique - le cinéma.

3 Lettres à lire dans le titre, image d'un nuage qui envahit l'écran, «smoke » connote également le processus de transformation et de réécriture de la nouvelle adaptée au format cinématographique. Le film se délecte en effet des multiples acceptions de « smoke » comme en atteste la lecture du scénario, dans lequel Paul Auster privilégie le rôle de la parole pour donner de l'épaisseur à des personnages doués d'une faconde parfaitement signifiée par l'expression idiomatique «to blow smoke»- raconter des salades. Le scénario ajoute avec ironie l'acte de fumer à la fin du repas qui scelle le contrat artistique entre Paul et Auggie, détail absent de la nouvelle, qui donne à voir la stratégie verbale adoptée par le scénariste. " "Smoke" exprime ce goût pour les bavardages qui ralentissent le rythme de la narration et soulignent le talent de conteur des personnages. Le découpage du film en cinq actes distincts, dédiés aux protagonistes qui les animent (1. Paul ; 2. Rachid ; 3. Ruby ; 4. Cyrus ; 5. Auggie), organise sa lente progression et trahit l'influence d'un scénario de facture dramatique, rappelant que Paul Auster s'était essayé à l'écriture de pièces de théâtre avant de connaître le succès grâce au roman - trois récits regroupés dans la Trilogie new-yorkaise (1987). ${ }^{3}$ Motif narratif et iconographique, "smoke " symbolise "l'intimité consubstantielle ${ }^{4}$ entre l'image et le texte dans l'écriture scénaristique de Paul Auster.

4 Du poids des mots au flux des images, le film permet à l'écrivain de s'initier à la réalisation cinématographique - aventure qu'il tentera en solitaire pour le tournage de Lulu on the Bridge (1998) et de The Inner Life of Martin Frost (2007). Le visionnage de Smoke demeure cependant une expérience singulière dans le cinéma américain; l'univers austerien se recompose à l'écran comme s'il existait au-delà des livres un horizon où se rencontrent le mot et l'image. La nouvelle et le film ont en commun de se nourrir de l'interpénétration des arts qu'ils font se croiser. L'appareil photo volé par Auggie constitue la pierre angulaire de cette association: l'expérience photographique et artistique d'Auggie évoque par métaphore le processus de création artistique à l'origine de Smoke. Elle suggère la relation de l'écrivain au réel et aux autres arts, une association reflétée par l'intertextualité qui travaille le matériau filmique.

5 La mise en scène de Smoke, qui souligne la transformation à l'œuvre dans le passage d'un code sémiotique à un autre (du verbal à l'iconique, de l'image fixe à l'image en mouvement), engage la réflexion sur la contiguïté entre les arts. Smoke convoque l'image de la fumée qui brouille la frontière entre les arts et les artistes devenus complices au fil du temps: la relation d'amitié entre l'écrivain en mal d'inspiration (Paul Benjamin) et le buraliste au talent de photographe (Auggie Wren) illustre à l'écran la collaboration entre l'écrivain renommé et le réalisateur sino-américain. Si Auggie offre à l'écrivain un récit qu'il peut réécrire sous son nom, Wayne Wang s'associe à Paul Auster pour faire de Smoke une œuvre austérienne. 


\section{La collaboration à l'origine d'une œuvre austerienne}

6 La collaboration artistique à l'origine de Smoke prolonge l'ambiguïté fondatrice du processus de création dont est issue la nouvelle : «Just put it down the way I told it to you and you don't owe me a thing " (Auster, 1995, 156) explique Auggie à la fin du déjeuner au cours duquel il a raconté l'histoire dont le narrateur pourra s'inspirer afin d'écrire le récit qu'il est censé livrer au New York Times pour Noël. L'œuvre de collaboration ferait-elle disparaître l'instance auctoriale? La collaboration n'existe-telle que sous la forme d'une appropriation du discours de l'Autre?

7 Les Cahiers du cinéma condamnèrent l'attitude du réalisateur Wayne Wang qui se serait laissé dépouiller de son film en associant l'écrivain-scénariste à sa conception. ${ }^{5}$ Wayne Wang aurait mis sa caméra au service de la plume de Paul Auster, s'efforçant de « rendre à l'écrivain tout ce qui lui appartient et même plus: lui offrir le film, en sacrifice ou en étrenne ». ${ }^{6}$ Fidèles à la " politique des auteurs » théorisée à travers deux articles écrits respectivement par Alexandre Astruc en 1948 et François Truffaut six ans plus tard, les Cahiers du cinéma mettent en lumière la signature du réalisateur au détriment du travail collectif de l'équipe filmique. La posture auctoriale défendue par Alexandre Astruc magnifie le rôle du réalisateur derrière la caméra qu'il compare de manière métaphorique à un stylo (la caméra-stylo) tandis que François Truffaut s'attache à mettre en évidence les "thématiques » spécifiques qui font la différence entre un auteur et un "besogneux » du cinéma. ${ }^{7}$ Jean-Michel Durafour souligne les limites de cette approche trop centrée sur l'individualisation de l'auteur qui conduit au " déni de l'œuvre dans sa singularité d'œuvre ».8

8 La collaboration à l'origine de Smoke permet la rencontre de deux univers créatifs, qui se rejoignent dans la pratique d'un cinéma indépendant, infléchi par les expérimentations alors novatrices menées par Quentin Tarantino (Pulp Fiction, 1994), Jim Jarmusch (Mystery Train, 1994), Robert Altman (Short Cuts, 1994), Bryan Singer (The Usual Suspects, 1994)... Tous s'affranchissent des codes du spectaculaire promus par le cinéma hollywoodien, ${ }^{9}$ en renouvelant les conventions de la narration filmique: éclatement du récit, goût pour le bavardage, pauses de silence, dissémination de l'attention sur plusieurs protagonistes, concrétisent le désir d'indépendance de ces réalisateurs à l'égard des normes hollywoodiennes.

Devenu une figure de proue du cinéma indépendant après la réalisation de Chan is Missing (1982), Wayne Wang contribua à faire reconnaitre le cinéma asiatique aux ÉtatsUnis, expérience filmique dont il laisse l'empreinte dans Smoke (Lin, 1998, 184-85). Le réalisateur y introduit desplages de silence, qui ralentissent le rythme de la narration pour ouvrir un espace de méditation à l'intérieur du film. Jun Xing suggère que ces silences font partie d'une esthétique wangienne et qu'ils permettent au spectateur d'apprivoiser les émotions du film. ${ }^{10}$ Conversations filmées en temps réel donnent à voir «le vécu de la pensée et de la réflexion [qui] fait partie des répliques » (Bernas, 2005, 247). La discussion est traversée par des silences qui expriment le raisonnement et les émotions derrière les mots échangés.

10 Cette remarque confirme les commentaires de Paul Auster puisqu'il insiste sur l'aspect collaboratif du travail entrepris avec Wayne Wang, dont il précise que l'influence se lit jusqu'au niveau de la place centrale accordée à la parole. ${ }^{11}$ Toutes les étapes de la construction du film (casting, musique, montage, générique...) ont fait l'objet d'une concertation, que Wayne Wang a souhaité afficher à travers la juxtaposition des deux 
noms dans le générique - «l'indice le plus flagrant de la spécificité d'un film que l'on peut rarement réduire à un seul auteur » (Blumenfield, 2008, 11). Le générique, lettres bleues sur fond noir, se démarque par sa simplicité et pose de manière frontale la question de l'hybridité entre lettres et images. Le générique de Smoke se lit et se regarde, « tout texte à lire est aussi texte à voir $»^{12}$ nous rappelle Roger Odin, suggérant la porosité des univers créatifs qui rassemblent Paul Auster et Wayne Wang.

11 Non synchronisée à l'image d'un écran noir où s'inscrivent les noms des réalisateurs puis des acteurs, la bande son ouvre le film par des bruits de fond qui invitent le spectateur à imaginer des rues animées par des jeux d'enfants et des conversations amicales. Une radio beugle les commentaires vifs d'un match de baseball au moment où le rideau noir se lève enfin sur une image de carte postale, plan fixe animé par un train qui traverse le champ, dessinant une frontière géographique entre le premier plan et la ligne de gratte-ciels qui se dessine à l'horizon, sur l'île de Manhattan. Des conversations enflammées envahissent progressivement l'espace sonore et créent une cacophonie qui sort le spectateur de la léthargie dans laquelle l'image l'avait installé, stratégie sonore utilisée à chaque changement de tableau, permettant une « entrée initiatique » dans la fiction selon l'expression d'Alain Arnaud. ${ }^{13}$

Les voix prennent corps avec un changement de lieu qui vise à ancrer le récit dans un décor minimaliste : à la vue panoramique se substitue l'intérieur du bureau de tabac où se déroule la première scène. Le rétrécissement de l'espace a pour effet de souligner l'artifice théâtral dans la mise en scène : le dialogue s'inscrit dans la durée cependant que le plan fixe rassemble les individus dans un huis clos (Prédal, 2007, 178). Cette mise en scène traduit le style de Wayne Wang, qui efface de manière caractéristique premier plan et arrière-plan. ${ }^{14}$ La caméra ne s'anime que pour suivre l'agitation des hommes qui se répondent avec virulence en écho aux équipes de baseball dont ils commentent les choix stratégiques. Les individus sont caractérisés par leur discours, une qualité oratoire qui les distingue dans une vie de quartier géographiquement éloignée des grands ensembles de Manhattan et restreinte à un carrefour de Brooklyn. Seuls les faits exceptionnels qu'ils peuvent se targuer de rapporter leur permettent de se faire remarquer.

$13 \mathrm{Au}$ milieu de ces bavards dont les discussions se focalisent habituellement sur le baseball, le personnage de Paul Benjamin met en abyme la représentation d'une identité masculine définie non par l'action, mais par l'aisance verbale, alors qu'il joue de manière ostentatoire la figure de l'écrivain. Il se distingue au milieu de cette assemblée masculine en racontant une anecdote sur Walter Raleigh, qui introduisit avec succès le tabac à la cour d'Elisabeth I où il fut traité en favori. Tout en manipulant amoureusement le cigare qu'il s'apprête à fumer, Paul distrait son auditoire comme Walter Raleigh amusa la reine en se proposant de calculer le poids de la fumée. L'homme campe avec un plaisir visible la fonction du conteur, placé au centre d'une scène qu'il occupe verbalement et domine physiquement, en contrepoint des personnages dont les visages demeurent hors champ et les voix désincarnées.

14 Si les aventures de l'explorateur anglais s'écoutent de la bouche du conteur dans Smoke, le nom de Walter Raleigh (1552-1618) est invoqué dans les romans de Paul Auster pour faire naître des images du Nouveau Monde..$^{15}$ Dans Leviathan, la référence à Walter Raleigh est utilisée pour caractériser le personnage de Sachs qui se targue d'avoir le poète pour ancêtre. Le narrateur souligne avec ironie la culpabilité de l'aïeul: « He [Sachs] was fond of saying that a poet was responsible for bringing his mother's family 
to Boston, but that was only a reference to Sir Walter Raleigh, the man who introduced the potato to Ireland and hence had caused the blight that occurred three hundred years later " (Auster, 1992, 24). Tissés de digressions qui lui permettent de dépasser l'opposition entre oralité et écriture, les romans de Paul Auster fournissent le matériau brut qu'une écriture scénaristique permet d'oraliser. À la différence du romancier dont l'art est d'écrire de belles lettres, l'écrivain confie volontiers que sa pratique littéraire relève du conte - «I tend to think of myself more as a storyteller than a novelist » (cité dans Brown, 2007, 33). La référence à Walter Raleigh souligne les références littéraires d'une écriture scénarisée et s'inscrit dans le jeu intertextuel qui informe le processus de création chez Paul Auster. ${ }^{16}$ Smoke se nourrit du jeu qui se noue entre l'art de la parole et celui de l'écriture, contiguïté artistique incarnée par le personnage de Paul Benjamin.

L'écrivain trouve dans la parole un mode d'action qui lui permet de sublimer sa panne d'écriture; son jeu d'acteur nous invite à définir l'action du film en termes de verve plutôt que de gestes. Le bavardage facile caractérise également le personnage d'Auggie lorsqu'il retrace l'aventure malheureuse de l'écrivain en crise d'inspiration depuis le décès accidentel de son épouse, tuée par une balle perdue lors d'un hold-up qui eut lieu dans le quartier. Un lent travelling avant resserre le cadre sur le visage d'Auggie dans une nouvelle tentative de dramatisation du verbe : la parole s'écoute et se regarde de même que le texte du générique se lit et se voit. Les réalisateurs ne cessent de jouer de la transmédialité du récit: l'écriture devient parole et image selon les modalités de l'adaptation déployées à l'écran.

16 Smoke croise les thématiques austeriennes, qui prolongent l'univers littéraire de l'écrivain à l'écran tout en illustrant l'interpénétration de son écriture et du cinéma. ${ }^{17}$ Figure fondamentale de l'écriture austérienne, la "coïncidence » est évoquée dans le film au niveau de la bande sonore par des bruits hors champ, qui rappellent l'existence d'un monde extérieur imprévisible et incontrôlable. Sirènes de police et coups de klaxon percent le discours d'Auggie, menacent de l'interrompre à tout moment, alors qu'il raconte la mort accidentelle d'Eileen. La bande sonore rappelle le jeu de hasard qui fut à l'origine de la tragédie, liée à un concours de circonstances malheureux. Auggie envisage d'autres possibilités - un peu de retard ce jour-là, une conversation un peu plus longue... auraient permis de sauver la vie de la jeune femme et de l'enfant qu'elle portait. François Gavillon explique que les romans de Paul Auster font de la « coïncidence » un ressort dramatique fréquent :

Parce que la coïncidence est à l'exacte croisée d'un monde déterministe et d'un monde chaotique. La répétition de l'événement ou sa superposition avec un autre donne l'intuition d'un ordre, d'une syntaxe fatidique. Mais en même temps, son caractère incongru, imprévisible et partant, inexplicable, pointe vers un univers chaotique (Gavillon, 2000, 126).

17 La coïncidence, ressort narratif utilisé dans le film comme dans les romans, est représentée avec justesse par ce carrefour dont Auggie prend des clichés quotidiens, espace de rencontres imprévues que le film met en scène. Auggie invite Paul à regarder sa collection de photos en noir et blanc et à reconstruire le récit d'un passé oublié en observant l'image du même coin de rue se répéter de cliché en cliché. L'image du carrefour illustre par autant de prises de vues le procédé narratif de la coïncidence, correspondant à l'image "d'un modèle réduit de l'œuvre elle-même qui se trouve incluse en elle pour la reproduire partiellement ou totalement » (Brugière et Topia, 2000 , 18). Cette mise en abyme ouvre le film à un questionnement artistique sur 
l'appropriation du réel dans la photographie et dans le processus créatif de l'écriture chez Paul Auster.

\section{Un art à la rencontre du réél : l'expérience photographique d'Auggie}

Un gros plan s'attarde sur les mains de Paul qui feuillette les albums avec indifférence ; les mêmes photos semblent se reproduire d'une page à l'autre, soulignant l'architecture orthogonale d'un carrefour traversé par quelques figures solitaires. L'originalité de l'œuvre photographique d'Auggie repose sur la dialectique de la différence et de la répétition qui la structure. La subjectivité du photographe est absorbée par le principe de répétition qui transforme l'expérience artistique en activité mécanique, impersonnelle. Le film souligne la distance entre Auggie et l'appareil photographique qu'il pose sur un trépied et déclenche à distance pour remplir sa mission quotidienne avec le « maximum d'objectivité dans l'appropriation subjective du réel ». ${ }^{18}$

Bien que l'auteur s'efface derrière l'exercice de style, la scène permet à Auggie de changer de statut dans la fiction; il incarne à son tour la figure de l'artiste, défini par le style conceptuel de ses photos qui opèrent un double décrochement narratif au sein du film. Les images ancrent la fiction dans le réel que l'appareil photographique prétend enregistrer sans mise en scène et ouvrent le film à un discours théorique sur la fonction de l'art. Auggie accompagne d'un commentaire en voix off la lecture des images; il transforme son travail en une affaire de mots comme il insiste sur les détails visuels qui doivent retenir l'attention de Paul et du spectateur : "You'll never get it if you don't slow down my friend. I mean you're going too fast, you're hardly looking at the pictures. They're all the same but each one is different from the other one ».

Le film adopte un rythme contemplatif qui permet de souligner l'acuité mécanique de l'œil photographique : la monotonie quotidienne inscrite dans la répétition du même plan à l'écran fait ressortir les différences d'ambiance entre les jours de semaine et les week-ends, les tonalités plus grises des matins pluvieux ou plus claires des journées ensoleillées... Les fondus-enchaînés relient les photographies pour construire une fiction du passé dans laquelle Paul finit par se laisser entraîner en reconnaissant la femme aimée sur l'une d'entre elles. La caméra zoome sur la silhouette de la défunte, avant de s'immobiliser et d'enfermer Paul dans la douleur d'une émotion indicible. Pour André Bazin, "les virtualités esthétiques de la photographie résident dans la révélation du réel » (Bazin, 1958, 16), ce qui confère à la scène une valeur épiphanique. Elle aide non seulement à caractériser le personnage de Paul, dont l'histoire personnelle est illustrée par le flash-back photographique, mais permet encore d'exposer sa relation au passé sous le sceau de la nostalgie. Alors qu'il contemple les photos avec plus d'attention et découvre les subtilités mentionnées par Auggie, les silhouettes fantomatiques prises au piège du papier photographique semblent s'animer. Les fondus enchaînés leur prêtent vie, faisant apparaître au jour de la conscience autant de souvenirs que de sentiments refoulés. "La photographie a quelque chose à voir avec la résurrection" (Barthes, 1980, 129) explique Roland Barthes dans La Chambre claire alors qu'il est lui-même saisi par le désir de retrouver l'image de sa mère telle qu'il s'en souvient parmi des photographies d'elle. «Est-ce que je la reconnaissais? » se demande-t-il, bouleversé par ce désir que l'image a fait renaître : «Dans ce désert morose, telle photo, tout d'un coup, m’arrive: elle m'anime et je 
l'anime. C'est donc ainsi que je dois nommer l'attrait qui la fait exister: une animation » (Barthes, 39).

21 La scène se rejoue dans Smoke au moment où Paul prend conscience du regard amoureux qu'il continue de poser sur la silhouette dont il a reconnu instinctivement la posture et l'attitude. L'animation dont parle Roland Barthes est faite "de désir, de répulsion, de nostalgie, d'euphorie » (Barthes, 41), autant de sensations et d'émotions endormies dans le corps de l'époux endeuillé, qui redécouvre un ancien désir face au cliché du souvenir. ${ }^{19}$ Le film reconstruit un morceau de mémoire oubliée alors que la caméra adopte le point de vue subjectif de Paul, s'approche de la femme aimée au moyen d'un zoom qui fait remonter à la surface de la conscience le souvenir d'un désir. 20

La fin de la séquence renvoie les deux artistes à leurs occupations respectives, deux modes d'expression qui nourrissent l'ambiguïté entre le réel et le fictionnel dans la nouvelle de Paul Auster. Les personnages appréhendent le monde environnant à travers l'écriture ou la photographie, deux arts de la solitude qui font naître une complicité amicale entre les deux hommes. Les prises de vues du carrefour symbolisent l'espace romanesque de Paul Auster, décrit par Annick Duperray comme "à l'image de son univers new-yorkais, au croisement de l'anonymat de la rue et de l'intimité de ceux qui la peuplent, au point d'intersection entre la parabole et l'histoire personnelle » (Duperray, 2003, 18). Le carrefour symbolise le point d'intersection entre une histoire collective et des destinées individuelles dans des photographies qui se présentent aussi comme un lieu de rencontre entre le présent et le passé, le visible et l'invisible, Paul et Auggie.

Le film dramatise le regard porté sur les photos à travers un point de vue subjectif qui brouille la distinction entre réalité et fiction. L'adaptation filmique transforme l'expérience photographique d'Auggie, car elle en souligne l'appropriation subjective à travers le regard de Paul. Seul l'acte de filmer les photographies permet de reconstruire l'instant épiphanique qui n'est d'ailleurs pas mentionné dans la nouvelle. Cet écart est dû aux possibilités ouvertes par les propriétés du film et remarquées ici par Jean-Pierre Esquenazi :

Le film se donne comme une mémoire, exhibant les principes selon lesquels le temps vécu se constitue en mémoire. Principe de dissociation du présent, principe d'appropriation subjective des représentations (caméra subjective), principe de conservation du passé en soi (Esquenazi, 1994, 132).

Dans la nouvelle, la photographie est le point de départ d'un travail imaginaire, un premier acte d'écriture pour l'écrivain qui se surprend à inventer des vies aux silhouettes observées - " as if I could imagine stories for them, as if I could penetrate the invisible dramas locked inside their bodies " (Auster, 1995, 152).

L'insertion des photographies dans la trame narrative du film fait encore ressortir l'intérêt de Paul Auster pour les autres arts, exprimé par sa participation au tournage de Smoke comme par ses références au travail entrepris en collaboration avec Sophie Calle, qui lui sert de modèle pour la création du personnage de Maria dans Leviathan.Auggie se plaît à exhiber chacune des pages de son œuvre photographique, dont la pratique ritualisée n'est pas sans rappeler les expériences menées par l'artiste française dans les rues de New York. ${ }^{21}$ Dans Leviathan, Paul Auster utilise le champ sémantique du regard pour commenter les qualités artistiques de Maria, autant de mots qui peuvent s'appliquer à décrire l'œuvre de Sophie Calle et d'Auggie : «Her subject 
was the eye, the drama of watching and being watched, and her pieces exhibited the same qualities one found in Maria herself : meticulous attention to detail, a reliance on arbitrary structures, patience bordering on the unendurable » (Auster, 1992, 63). ${ }^{22}$ Le film rejoint alors le roman dans son utilisation de l'appareil photographique comme une "source de déréalisation ", ${ }^{23}$ qui permet à Auggie de porter un discours critique sur l'œuvre qu'il expose en citant Shakespeare: "Tomorrow and tomorrow and tomorrow... Time creeps on its petty pace... ». Clichés saisis sur le vif par l'appareil, les photographies d'Auggie se présentent comme l'enregistrement d'un quotidien dont elles sont la trace. L'expérience individuelle et impersonnelle de la photographie devient universelle grâce à la conjonction du fondu enchaîné qui lui confère une dimension phénoménologique: les photographies insérées dans le montage du film manifestent le temps qui passe, elles fabriquent une mémoire du passé comme elles symbolisent la durée infinie.

L'œuvre photographique d'Auggie n'a pas seulement valeur intertextuelle, elle illustre par la métaphore le processus de création qui nourrit l'acte d'écriture de Paul Auster. "Image qui se donne à voir comme si elle était le réel même " (Caraion, 2003, 42), la photographie incite à une réflexion sur les rapports entre le réel et l'illusion, ainsi que sur son appropriation par la mise en images dans le film.

\section{L'appropriation comme processus de création artistique}

L'étude de Smoke nous invite à retrouver ces éléments qui, tirés d'« Auggie Wren's Christmas Story ", sont disséminés dans le film et soulignent l'effort d'appropriation qui accompagne la réécriture de la nouvelle par le cinéma. Le cadre photographique peut se lire comme métaphore de la forme narrative courte de la nouvelle, suggérant les aventures qui se déroulent dans un espace hors-champ. Les échos multiples entre la séquence finale et les actes précédents montrent que l'écrivain a scénarisé sa nouvelle en prolongeant les trames narratives du récit court. Adaptée dans le générique de fin, la nouvelle renvoie aux anecdotes qui ont ponctué le film: Auggie voit l'adolescent dérober un magazine dans sa boutique au cours de l'Acte 1 tandis qu'il s'élance après le voleur dont il a remarqué les agissements dans le générique; Auggie découvre dans le portefeuille du voleur (Roger Goodwin) une adresse située dans la cité de Boerum Hills, d'où vient également Rachid, le jeune garçon qui apparaît de manière fortuite dès l'Acte 1 et retient Paul Benjamin par le bras avant qu'il ne traverse la route devant les roues d'un camion; l'absence de figure paternelle dans la vie de Rachid rencontre un écho saisissant sur les photos de Roger Goodwin, entouré uniquement de sa mère et d'un frère plus jeune; Ruby, l'ancienne petite amie d'Auggie qui vient lui annoncer sa possible paternité d'une jeune femme de vingt ans dans l'Acte 3, est borgne comme Granny Ethel est aveugle...

Les références aux actes précédents trahissent le récit qu'Auggie vise à ancrer dans le monde réel, pour révéler un imaginaire de conteur, judicieusement illustré par le rêve mentionné dans le refrain de la chanson interprétée par Tom Waits ("You're innocent when you dream »). La musique se superpose à des images dont le contraste noir et blanc évoque la couleur de l'encre sur le papier de la machine à écrire qui apparaît en gros plan à l'écran. L'acte d'écrire rejoint l'acte de filmer dans cette image qui se lit comme un aveu de fiction et présente les modalités de l'appropriation d'un art par un autre : le 
nom d'Auggie Wren côtoie celui de Paul Benjamin sur la page blanche, associant la parole du conteur à la dactylographie de l'écrivain, sous l'œil de la caméra des réalisateurs. Les lettres bleues du générique d'ouverture reviennent se superposer au récit en images, créant un dédoublement de l'image à lire, qui suggère l'interpénétration des arts à l'origine de Smoke.

Le film se conclut sur une double adaptation de la nouvelle : la première partie exhibe l'art de la parole en se focalisant sur l'expression faciale d'Auggie pendant qu'il retrace le Noël passé avec Granny Ethel et les événements qui l'ont conduit chez elle, tandis que la deuxième raconte la même histoire en empruntant les codes du cinéma muet, la parole laissant place à la chanson. L'effet de zoom utilisé pour filmer la bouche d'Auggie, qui finit par envahir l'écran dans un insert, fait disparaître l'homme derrière son discours comme par métonymie; Auggie n'existe qu'à travers les mots dont il abreuve Paul et le spectateur, absorbés par son récit et bercés par la musique de sa voix. La caméra s'approche de la bouche d'Auggie pour mieux recueillir son discours et se l'approprier dans une image qui évoque l'insertion d'un art dans un autre, thématique récurrente dans les romans de Paul Auster.

30 Auggie parle au passé, à la première personne, et son regard caméra rapproche son attitude de la confession. L'homme présente son acte de parole comme l'expiation d'une faute commise, un acte de rédemption, puisqu'il offre à Paul le récit d'une histoire qui permettra à l'écrivain de surmonter son blocage d'écriture. Le larcin confessé se meut en conte de Noël sous la plume de l'écrivain, qui va offrir sa version de l'histoire aux lecteurs du New York Times. A la fin de la séquence, le sourire ébloui d'Auggie trahit la jouissance du conteur qui a berné son auditoire tandis que Paul se découvre l'heureuse victime de l'illusion de la fiction et complimente le maitre : «Bullshit is a real talent, Auggie. To make up a good story, a person has to know how to push all the right buttons. I'd say you're up among the masters ". L'écrivain est sensible au pouvoir des mots que le conteur sait manipuler pour mieux faire se confondre le réel et l'illusion - dialectique illustrée par les photographies d'Auggie et incarnée par le personnage de Thomas Jefferson Cole.

Contraction de Thomas Jefferson, président américain de 1801 à 1809, et de Thomas Cole, célèbre peintre paysagiste américain du XIXe siècle, le nom souligne la double identité du jeune garçon qui le porte. Fondateurs d'un mouvement appelé l'école de la vallée de l'Hudson (Hudson River School), les tableaux de Thomas Cole célèbrent la grandeur sauvage des paysages américains en jouant des effets de lumière sur les masses rocheuses. ${ }^{24}$ Son art pictural prolonge la découverte des terres américaines situées à l'Ouest, politique d'exploration soutenue par le président Thomas Jefferson, et exprime le sentiment du sublime que des panoramas vertigineux pouvaient inspirer aux aventuriers animés par la mission d'une Destinée Manifeste. Dans Smoke, la référence au peintre dénonce le jeu des apparences orchestré par Thomas Jefferson Cole, qui se fait appeler Rachid quand il rencontre Paul Benjamin, dont il emprunte ensuite le nom pour se cacher d'un père qui l'a abandonné mais qu'il va retrouver Cyrus Cole (Acte 4). Le nom de Thomas Jefferson Cole connote une réalité que le jeune homme tente de dissimuler par l'invention d'une autre histoire et d'une autre identité, stratégie picturale à l'œuvre dans les peintures de Cole qui véhiculent une version idéalisée des paysages. La double paternité inscrite dans le nom de Thomas Jefferson Cole évoque un art construit sur une appropriation du réel qu'il vise à sublimer. Tel est peut-être le projet artistique que représente Smoke. 
32 Le personnage, interprété par un jeune acteur de couleur, incarne une diversité culturelle absente du scénario et proposée par Wayne Wang (Hooks, 1996, 137). L'origine afro-américaine $\mathrm{du}$ garçon et son nom aux connotations anglo-saxonnes (Thomas Cole était natif d'Angleterre) suggèrent une double appartenance culturelle qui n'est pas sans rappeler l'échange culturel et artistique entre un réalisateur originaire de Hong-Kong, Wayne Wang, et un écrivain new-yorkais, Paul Auster. La critique et écrivaine afro-américaine bell hooks souligne l'apport culturel du réalisateur, qui introduit une image métissée de Brooklyn, en rupture avec la vision «blanche» qui prévaut dans l'imaginaire américain. Le film ouvre l'espace de la représentation aux Afro-Américains, qui peuvent se réapproprier le quartier à travers l'image multiraciale proposée. ${ }^{25}$

\section{Conclusion}

"Smoke is something that is constantly changing in shape, and never fixed ", explique Paul Benjamin. La métaphore permet de décrire le parcours des personnages du film, qui se modifie au hasard des rencontres et des coïncidences que symbolisent avec justesse les photographies du carrefour collectionnées par Auggie. Smoke signifie cette fluctuation du sens entre l'image et la lettre, la littérature et le cinéma, autant de possibilités ouvertes par l'adaptation cinématographique. Les références intertextuelles abondent dans l'art de Paul Auster, pratiquant l'autocitation pour mieux souligner l'arbitraire du signe. Le nom de Thomas Cole n'est pas convoqué pour suggérer les mêmes valeurs dans Moon Palace ou dans Smoke, ce qui suggère la polysémie des signes permise par l'insertion des images dans l'écriture ainsi que par l'hybridité du mot et de l'image dans le film. Smoke se présente comme une réécriture qui met en relief les ressorts de l'écriture austérienne : les images se lisent comme des textes. Ainsi la cité de Boerum Hills, filmée en contre-plongée dans le générique de fin, laisse entrevoir des bouts de ciel entre les sommets d'immeubles identiques les uns aux autres et pourtant tous différents - quadrillage qui interroge le rapport entre l'art et le réel en reproduisant la dialectique présente dans les albums photographiques d'Auggie.

Produit d'une rêverie collective entre un écrivain et un réalisateur, rassemblés autour d'une adaptation qui permet aux arts de s'associer sur le mode de l'appropriation, Smoke devient synonyme de métafiction, un acte de narration dont le pouvoir de manipulation est symbolisé à l'écran par l'image de la bouche d'Auggie. Le récit d'une histoire s'échange contre la dégustation d'un repas dans une scène qui suggère la valeur marchande de l'art, objet de consommation et de possession, puisque le conte de Noël d'Auggie est réduit à une monnaie d'échange comme le film qu'il a inspiré. Si le rendez-vous littéraire et culinaire de l'écrivain et du conteur/photographe évoque l'arrangement dont ont pu profiter Paul Auster et Wayne Wang - l'un se faisant offrir un film, l'autre une histoire, il illustre également la création artistique comme un acte collectif, qui se nourrit du partage et de l'appropriation des idées de l'Autre.

De manière tout à fait surprenante et inattendue, les films ultérieurs de Paul Auster laissent penser que sa pratique filmique est plus visuelle que verbale. Lulu on the Bridge est une fable moins bavarde, où l'image supplée le texte - l'énigme policière au cœur du film s'éclaircit dans la scène finale au moyen du sonore et du visuel. L'héroïne fait son signe de croix car la sirène d'une ambulance vient de s'éteindre, signe que la mort a frappé l'homme blessé à l'intérieur, victime d'une balle perdue. Le film se comprend de 
manière rétrospective à la lumière de cette scène qui révèle l'intrigue comme le dernier songe du mourant. Si le nom de Paul Auster est à nouveau crédité dans le générique de The Center of the World (Le Centre du monde, Wayne Wang, 2002), le film est présenté comme une œuvre wangienne, caractérisée par des plans fixes silencieux, un récit discontinu, des séquences tournées caméra à l'épaule, une image digitale, créant une atmosphère intimiste dérangeante autour d'une intrigue sexuelle. La séquence de fin répète la séquence d'ouverture, comme pour souligner un refus de clôture narrative, qui souligne le désir d'indépendance de Wayne Wang à l'égard du système hollywoodien qu'il n'a jamais complètement intégré. ${ }^{26}$

Smoke a permis la rencontre de deux univers artistiques, qui s'enrichissent de l'assemblage culturel provoqué, en travaillant le matériau filmique pour qu'il entre dans une autre intertextualité filmique - celle que prolongera la collaboration renouvelée dans Blue in the Face (Paul Auster et Wayne Wang, 1995). Le film est une autre œuvre singulière, animée par des acteurs issus du cinéma indépendant (Jim Jarmusch, Lou Reed) et rythmée par les conversations quotidiennes qui les intéressent, autour du même coin de rue que dans Smoke. L'aventure cinématographique se poursuit de manière moins formelle sous l'impulsion de l'improvisation, expérimentation visuelle et narrative qui offre de nouvelles pistes de création à la croisée des arts.

\section{BIBLIOGRAPHIE}

ARNAUD, Alain, « Par où commencer : le générique, désir de récit ou récit de désir ? » dans Cahiers du CIRCAV, n 9, 1997.

AUSTER, Paul, Auggie Wren's Christmas Story, dans Smoke and Blue in the Face: Two Films, New York, Miramax Books, 1995, 151-56.

AUSTER, Paul, Leviathan, London, Faber and Faber, 1992.

AUSTER, Paul, Mr Vertigo, London, Faber and Faber, 1994.

AUSTER, Paul, Moon Palace, London, Penguin Books, 1989.

AUSTER, Paul, The New York Trilogy, London, Faber and Faber, 1987.

BARTHES, Roland, La Chambre claire, Note sur la photographie, Paris, Seuil, 1980.

BAZIN, André, « Ontologie de l'image photographique » dans Qu'est-ce que le cinéma ?, Paris, Les Editions du Cerf, 1958.

BELL, Hooks, "The Cultural Mix : an Interview with Wayne Wang”, Reel to Race : Race, sex and class at the Movies, New York, Routledge, 1996.

BENJAMIN, Walter, Illuminations, New York, Schocken Books, 1969.

BRUGIÈRE, Bernard et TOPIA André, dir., « Préface ", L'Art dans l'art : littérature, musique et arts visuels (monde anglophone), Paris, Presses de la Sorbonne Nouvelle, 2000, 7-22.

BERNAS, Steven, L'Écrivain au cinéma, Paris, L'Hamattan, collection « Champs Visuels », 2005. 
BOLTER, Trudy, « Noir into Black on White : Smoke by Paul Auster and Wayne Wang (1995) » dans Dominique Sipière et Gilles Ménégaldo , dir., Les Récits policiers au cinéma, Poitiers, La Licorne, 1999, 301-17.

BLUMENFIELD, Samuel, « Préface » dans Alexandre Tylski, dir., Les Cinéastes et leur générique, Paris, L'Harmattan, collection « Champs Visuels », 2008, 9-15.

BROWN, Mark, Paul Auster, Manchester, Manchester University Press, 2007.

BRUGIÈRE, Bernard et TOPIA, André, dir., L'Art dans l'art : littérature, musique et arts visuels (monde anglophone), Paris, Presses de la Sorbonne Nouvelle, 2000.

CARAION, Marta, Pour fixer la trace, Photographie, littérature et voyage au milieu du XIXe siècle, Genève, Droz, 2003.

DESPOIX, Philippe et Schöttler, Peter, dir., Siegfried Kracauer, penseur de l'histoire, Laval, Les Presses de l'Université de Laval, 2006.

DUPERRAY, Annick, Paul Auster, Les ambiguités de la négation, Collection « Voix américaines », Paris, Belin, 2003.

DURAFOUR, Jean-Michel, Hawks, cinéaste du retrait, Villeneuve d'Asq, Presses Universitaires du Septentrion, 2007.

ESQUENAZI, Jean-Pierre, Film, perception et mémoire, Paris, L'Harmattan, 1994.

GAVILLON, François, Paul Auster, gravité et légèreté de l'écriture, collection « Interférences », Rennes, Presses Universitaires de Rennes, 2000.

HAMAMOTO, Darrel Y. et Liu Sandra, dir., Countervisions : Asian American Film Criticism, Philadelphia, Temple University Press, 2000.

HUGHES, Gérard et Royot Daniel, dir., « La littérature anglo-américaine à l'écran»dans Cahiers et Documents 12, Paris, Didier Erudition, Juin 1993.

LAPSLEY, Robert et WESTLAKE, Michael, dir., Film Theory : an Introduction, Manchester, Manchester University Press, 1988.

LIN, Jan, Reconstructing Chinatown : Ethnic Enclave, Global Change, Minneapolis, University of Minesota Press, 1998.

MARCEL, Christine, « La question de l'auteur dans l'œuvre de Sophie Calle. Unifinished. » dans Sophie Calle, M'as-tu vue, Paris, Co-édition Centre Pompidou-Xavier Barral, 2003, 15-28.

MOTTET, Jean, L'Invention de la scène américaine : cinéma et paysage, Paris, L'Harmattan, collection «Champs Visuels », 1998.

ODIN, Roger, « L'entrée du spectateur dans la fiction », Théorie du film, Paris, Albatros, 1980.

PESSO-MIQUEL, Catherine, Toiles trouées et déserts lunaires dans Moon Palace de Paul Auster, Paris, Presses de la Sorbonne Nouvelle, 1996.

PRÉDAL, René, Cinéma sous influence, Paris, L’Harmattan, collection « Champs Visuels », 2007.

STRAUSS, Frédéric, « Smoke », Cahiers du cinéma nº 497, décembre 1995.

XING, Jun, Asian America through the Lens : History, Representations, and Identity, Lanham, Alta Mira Press, 1998. 


\section{NOTES}

1. Paul Auster multiplie les effets de réel en incluant dans la fiction l'idée que la nouvelle répond à une commande du New York Times. Il insiste sur l'aspect autobiographique dès l'incipit pour entretenir la confusion : «I heard this story from Auggie Wren. Since Auggie doesn't come off too well in it, at least not as well as he'd like to, he's asked me not to use his real name ». Paul Auster, "Auggie Wren's Christmas Story » dans Smoke and Blue in the Face: Two Films, New York, Miramax Books, 1995, 151.

2. La dernière phrase de la nouvelle ne mentionne pas l'acte de fumer: "I returned Auggie's smile with a smile of my own, and then I called out to the waiter and asked for the check ». Paul Auster, op. cit., 156. Le scénario ajoute avec ironie cet élément: " Auggie lights a cigarette; Paul lights a little cigar. They blow smoke into the air, still smiling at each other », transformant le mot en image. Ibidem, 150.

3. Paul Auster, The New York Trilogy, London, Faber and Faber, 1987. La trilogie est composée de : City of Glass (1985), Ghost (1986) et The Locked Room (1987).

4. «L'association d'un art à un autre implique tantôt des liens de contiguïté ou d'analogie, tantôt une intimité consubstantielle Ainsi la présence de la peinture dans la littérature peut d'abord se comprendre au niveau de l'intrigue, de la thématique, ou encore de résonances symboliques parfois accompagnées de réflexions sur l'art. » Bernard Brugière et André Topia, dir., " Préface ", L'Art dans l'art : littérature, musique et arts visuels (monde anglophone), Paris, Presses de la Sorbonne Nouvelle, 2000, 8.

5. Paul Auster devient visible contrairement aux scénaristes habituellement prisonniers de l'ombre. Dans la plupart des films, le «scénario n'acquiert aucune visibilité sur l'écran, aussi relègue-t-il son auteur à un rôle subalterne par rapport au metteur en scène ». Voir Gérard Hughes, Daniel Royot, «La Littérature anglo-américaine à l'écran»dans Cahiers et Documents 12, Paris, Didier Erudition, Juin 1993, 2.

6. Frédéric Strauss, «Smoke» dans Cahiers du cinéma n 497, décembre 1995, 62. L'auteur voit la collaboration des artistes sous un angle opportuniste : «Cette manière captieuse, illégitime, de présenter le film dit sa vraie nature : fantasme snob et naif qu'une vedette du monde littéraire fasse son cinéma. C'est à quoi s'applique Wayne Wang, avec une humilité de caudataire ».

7. Pour une perspective critique sur l'évolution de la politique des auteurs, voir Robert Lapsley et Michael Westlake, Film Theory : an Introduction, Manchester, Manchester University Press, 1988, 105-28.

8. Jean-Michel Durafour déplore que l'œuvre soit jugée à l'aune de son auteur: "Le film ne devient jamais autonome, puisqu'il est jugé en fonction de son auteur ». Jean-Michel Durafour, Hawks, cinéaste du retrait, Villeneuve d'Asq, Presses Universitaires du Septentrion, 2007, 25.

9. Voir les pages consacrées à la « stratégie du spectaculaire » dans Nolwenn Mingant, Hollywood à la conquête du monde, marchés, stratégies, influences, Paris, CNRS Editions, 2010, 88-91.

10. "Constant 'spaces' of silence in Smoke, which is often assumed to be his signature narrative structures, leave the audience to deal with different kinds of emotions. » Jun Xing, Asian America through the Lens: History, Representations, and Identity, Lanham, Alta Mira Press, 1998, 202.

11. DVD (TF1 Vidéo, 2000) Paul Auster explique: «He [Wayne Wang] wanted the words to be the core of the film ». Il présente le film comme une collaboration dont sont imprégnées toutes les étapes de la réalisation: «a collaborative effort: the casting, the music, the editing, the credits, everything you see in the film is really the result of a two-man collaboration. It was Wayne who at some point during the filming insisted the film have the credits 'filmed by Wayne Wang and Paul Auster'».

12. «Tout texte à lire est aussi texte à voir ; un texte écrit, c'est d'abord une image, ou plutôt un ensemble d'images (les lettres, comme unités typographiques). » Roger Odin, "L'entrée du spectateur dans la fiction », Théorie du film, Paris, Albatros, 1980, 198. 
13. L'auteur précise : «Le son permet l'entrée initiatique du spectateur dans la fiction par un affect qui, à partir du générique, se réplique dans le film à l'instar du signifiant auquel il se rattache ». Alain Arnaud, «Par où commencer : le générique, désir de récit ou récit de désir?» dans Cahiers du CIRCAV, $\mathrm{n}^{\circ}$ 9, 1997, 142.

14. Le réalisateur explique: «There's no foreground or background. I kind of used the [principle] as my guidance for looking at the world. A non emphasizing camera position ». Jun Xing, op. cit., 150 .

15. Sir Walter Raleigh est mentionné dans les ouvrages suivants: Paul Auster, Moon Palace, London, Penguin Books, 1989; Paul Auster, Leviathan, London, Faber and Faber, 1992; Paul Auster, Mr Vertigo, London, Faber and Faber, 1994. Smoke se nourrit de l'intertextualité qui caractérise toute l'œuvre austérienne: "Les textes d'Auster ont une origine intérieure et un déploiement réflexif. Ils trouvent naissance dans l'intertextualité avec la littérature américaine, dans l'élément autobiographique, et se développent dans l'intertextualité interne, dans le sous-texte métafictionnel et dans le schéma symétrique qui met en regard auteur et personnage, réalité et fiction ». François Gavillon, Paul Auster, Gravité et légèreté de l'écriture, collection «Interférences ", Rennes, Presses Universitaires de Rennes, 2000, 178.

16. Trudy Bolter explique: «The 'ideal spectator' for Smoke is a reader of Paul Auster's work, able to put together the above-mentioned clues - and others as well - capable, indeed, of becoming a literary detective, for he is familiar with every detail of Paul Auster's work as a fan of Sherlock Holmes or Arsène Lupin, knowing all the nooks and crannies of his favourite mental furniture. The Austerian film spectator is thus a double of the confirmed Austerian book reader and, indeed, watching the film is perhaps an experience as close as the cinema can provide». Trudy Bolter, «Noir into Black on White: Smoke by Paul Auster and Wayne Wang (1995)» dans Dominique Sipière et Gilles Ménégaldo, dir., Les Récits policiers au cinéma, Poitiers, La Licorne, 1999, 311.

17. The Book of Illusions (2002) illustre cet aspect alors que l'intrigue conduit Mr Zimmer, écrivain accaparé par l'écriture d'un essai sur Hector Mann, à retrouver le réalisateur oublié du cinéma muet.

18. Siegfried Kracauer évoque la technique du « déclencheur automatique » «qui représenterait non pas un objectivisme aberrant, mais en quelque sorte le maximum d'objectivité dans l'appropriation subjective du réel». Peter Schöttler, «L'historien entre objectivisme et subjectivisme" dans Philippe Despoix et Peter Schöttler, dir., Siegfried Kracauer, penseur de l'histoire, Laval, Les Presses de l'Université de Laval, 2006, 85.

19. Marta Caraion rapporte que le même type d'expérience fut mené par Ernest Lacan: «parcourir des photographies jusqu'à trouver l'image du souvenir, de l'émotion, celle que l'on reconnaît parmi toutes comme répondant à un ancien désir ». Marta Caraion, Pour fixer la trace, Photographie, littérature et voyage au milieu du XIXe siècle, Genève, Droz, 2003, 175.

20. Walter Benjamin suggère que la caméra cinématographique ouvre l'œil du spectateur en donnant à voir un espace qu'il ne voit habituellement pas : "Evidently a different nature opens itself to the camera than opens to the naked eye - if only because an unconsciously penetrated space is substituted for a space consciously explored by man. [...] Here the camera intervenes with the resources of its lowerings and lifting, its interruptions and isolations, its extensions and accelerations, its enlargements and reductions. The camera introduces us to unconscious optics as does psychoanalysis to unconscious impulses ». Walter Benjamin, Illuminations, New York, Schocken Books, 1969, 236-37.

21. La collaboration entre Paul Auster et Sophie Calle nourrit une création artistique originale fondée sur un échange de pratiques : «A la suite d'un projet de film inabouti sur l'artiste Sophie Calle, auquel il a travaillé, Paul Auster emprunte la réalité de l'auteur Sophie Calle dans sa fiction Leviathan. Il invente le personnage de l'artiste Maria, qui plagie sept œuvres de l'artiste Sophie Calle (Suite vénitienne, La Garde-robe, Le Strip-tease, La Filature, l'Hôtel, Le Carnet d'adresses et Le Rituel 
d'anniversaire), mais ajoute deux œuvres fictionnelles. En tant qu'auteur, elle se retrouve donc personnage de la fiction d'un autre et auteur potentiel d'une œuvre fictionnelle. Puis elle opère un palimpseste pur et simple en reproduisant les passages de Leviathan, et corrige la fiction de Paul Auster en fonction de sa réalité. Elle reprend ainsi sa place d'auteur en entourant les passages de Leviathan en rouge, en les biffant ou en les réécrivant. La subtilité réside ici dans le fait qu'elle réalise un palimpseste d'une œuvre qui est déjà un palimpseste de la sienne, où elle fait renaître l'auteur réal en effaçant la fiction. » Christine Marcel, « La question de l'auteur dans l'œuvre de Sophie Calle. Unifinished. » dans Sophie Calle, M'as-tu vue, Paris, Co-édition Centre Pompidou-Xavier Barral, 2003, 26.

22. Auggie et Maria voient leur art comme une mission personnelle: «Maria was an artist, but the work she did had nothing to do with creating objects defined as art. Some people called her a photographer, others referred to her as a conceptualist, still others considered her a writer, but none of these descriptions was accurate, and in the end I don't think she can be pigeonholed in any way. [...] Living always came first, and a number of her most time-consuming projects were done strictly for herself and never shown to anyone. " Auster, Leviathan, op. cit., 60.

23. François Gavillon explique que «Le paradoxe fondamental qui retient d'abord l'attention de l'auteur, c'est que l'observation est source de déréalisation ». François Gavillon, op. cit., 177.

24. Jean Mottet, L'Invention de la scène américaine : cinéma et paysage, Paris, L'Harmattan, collection "Champs Visuels ", 1998, 18. Les tableaux de Thomas Cole sont également mentionnés dans Moon Palace (Paul Auster, Moon Palace, op. cit., 108); la toile mentionnée dans le roman est une œuvre encombrée de silhouettes humaines, qui participe «d'une subversion de l'image idéalisée de l'Amérique ». Voir les commentaires de Catherine Pesso-Miquel, Toiles trouées et déserts lunaires dans Moon Palace de Paul Auster, Paris, Presses de la Sorbonne Nouvelle, 1996, 52-53.

25. bell hooks ajoute: "What's interesting is that you don't invoke the conventional, sort-ofwhite culture of Brooklyn that has come to stand in the public imagination of what real Brooklyn culture is. You see so many ethnicities, and across so many immigrant groups, a consistent feeling of passion about the space, about the environment, about Brooklyn ». bell hooks, "The Cultural Mix: an Interview with Wayne Wang ", Reel to Race: Race, sex and class at the Movies, New York, Routledge, 1996, 133.

26. Produit et distribué par Miramax (filiale des studios Walt Disney depuis 1993), Smoke éloigna Wayne Wang des thématiques culturelles privilégiées par le film ethnique et lui permit de se faire remarquer par les studios hollywoodiens. «Since Smoke, Wang and Hollywood have continued to dance around each other. While Smoke was still in production in late 1994, movie industry magazines began reporting on Wang's upcoming projects. Hollywood Pictures signed Wang to direct The Kitchen God's Wife. [...] ». Darrel Y. Hamamoto et Sandra Liu, dir., Countervisions: Asian American Film Criticism, Philadelphia, Temple University Press, 2000, 96.

\section{RÉSUMÉS}

L'adaptation de la nouvelle « Auggie Wren's Christmas Story » (1990) réunit l'écrivain Paul Auster et le réalisateur Wayne Wang autour d'un projet qui leur permit de croiser leurs pratiques artistiques dans un travail de collaboration. Cet article prolonge le questionnement artistique qui sous-tend le récit de la nouvelle en interrogeant les modalités de l'appropriation du réel par d'autres arts. La première rencontre entre Paul Auster et le cinéma met en relief les points de 
convergence entre écriture verbale et écriture cinématographique, évoqués autour de la double figure de l'écrivain et du conteur/photographe dans le film et dans la nouvelle.

The adaptation of the short story entitled "Auggie Wren's Christmas Story" (1990) gathered the writer Paul Auster and the director Wayne Wang around a film project that allowed them to share their artistic practices in a collaborative work. This paper examines the question that underpins the narrative of the short-story by grappling with the appropriations of the real in other artistic modes. Paul Auster's first encounter with the cinema points out the convergence between aural writing and cinematic writing, evoked through the double figure of the writer and that of the tale-teller/photographer in both the film and the short story.

\section{INDEX}

Mots-clés : Paul Auster, Wayne Wang, cinéma indépendant, photographie, interpénétration des arts.

Keywords : Paul Auster, Wayne Wang, independent cinema, photography, interpenetration between the arts.

Thèmes : Hors-thème

\section{AUTEUR}

\section{DELPHINE LETORT}

Université du Maine 